\title{
A Plethysmographic Method for Assessment of Lung Function in Mechanically Ventilated Very Low Birth Weight Infants
}

\author{
K. E. EDbERG, K. SANDBERG, A. SILBERbERG, B. A. SJÖQViST, B. EKSTRÖM-JODAL, AND \\ O. HJALMARSON \\ Department of Anesthesiology and Intensive Care and Department of Pediatrics, Gothenburg University, and \\ Research Laboratory of Medical Electronics, Chalmers University of Technology, Gothenburg, Sweden
}

\begin{abstract}
We have developed and tested a plethysmographic method for assessment of lung function in mechanically ventilated very low birth weight infants during intensive care. Information about the mechanics of the respiratory system is obtained from the respiratory flow as measured by volume displacement plethysmography and from airway pressure measured in the artificial airway. Data on lung volumes, ventilation, and distribution of ventilation is obtained simultaneously by combining the respiratory flow measurements with nitrogen concentration analyses of the respiratory gas. No significant differences were found when the estimations of mechanical parameters and FRC were compared with reference methods and when determinations of the same parameters were repeated in the same subjects. The plethysmograph was shown to be safe and convenient to use, even in studies lasting several hours. (Pediatr Res 30: 501-504, 1991)
\end{abstract}

\section{Abbreviations}

$\mathrm{FiO}_{2}$, fraction of inspired oxygen

FRC, functional residual capacity

Assessment of lung function in very low birth weight infants with severe respiratory disease is complicated by their small size and by the fact that they are generally very fragile. The intensive care situation also makes access to these infants difficult. One important problem relating to lung function studies in intubated newborns is leakage of respiratory gas around the uncuffed endotracheal tube that is normally used in neonatal intensive care. If respiratory flow is measured as the flow that passes through the endotracheal tube, leakage around the tube creates a source of error. One way of avoiding this problem is to use a body plethysmograph for flow measurement, assuming this technique can meet the necessary requirements.

The aim of this study was to develop and test a system for assessment of lung function, using modified body plethysmography in mechanically ventilated preterm newborn infants during intensive care. We intended to measure FRC, gas mixing efficiency, total and alveolar ventilation, and mechanics of breathing.

Received January 9, 1991; accepted April 24, 1991

Correspondence: Karl Erik Edherg MD Department of Pediatric Anesthesia and Intensive Care, Children's Hospital, S-416 85 Gothenburg, Sweden.

Supported by Grants No. 5703 and 7543 from the Swedish Medical Research Council.

\section{MATERIALS AND METHODS}

The recording system consisted of a face-out volume displacement body plethysmograph, an endotracheal tube connector for alternate use of two ventilators and with ports for measurements of airway pressure and nitrogen concentration.

Body plethysmograph. The plethysmograph is made of $12-\mathrm{mm}$ Perspex (Svenska ICI, Gothenburg, Sweden) with maximum inner dimensions of $485 \times 275 \times 125 \mathrm{~mm}$ (Fig. 1). The inner volume is $14.5 \mathrm{~L}$. These dimensions were chosen so that the plethysmograph could be used inside an incubator, to preserve the environmental conditions for the infant during the study. The plethysmograph consists of three separate parts: a bottom plate, a cover hood, and a soft circular cuff to be placed and sealed around the infant's head and face. The three parts can easily be attached to one another and can also be easily and rapidly disconnected when necessary. In the cover hood, there are airtight exits for cables and catheters and a connection for a pneumotachograph. The face cuff consists of a circular soft plastic bag filled with small polythene particles about $1 \mathrm{~mm}$ in diameter. The connections between the three different parts are kept airtight by means of rubber seal plates.

Flow measurements. Flow is measured by a pneumotachograph (Fleisch no. 1, Siemens-Elema, Gothenburg, Sweden), which is mounted into the box wall. It is used unheated, because the temperatures inside the box and in the incubator are approximately the same. The pneumotachograph is connected to a differential pressure transducer (Elema-Schönander EMT 32, Siemens-Elma).

Airway pressure and nitrogen concentration. A special endotracheal tube connector was built to connect two ventilators to the endotracheal tube, to measure airway pressure, and to permit sampling of gas for measuring nitrogen concentrations at the proximal end of the endotracheal tube (Fig. 2). A polyethylene catheter (length $770 \mathrm{~mm}$, inner diameter $1.1 \mathrm{~mm}$ ) passing through a port in the endotracheal tube connector was connected to a pressure transducer with a small measuring chamber and a stiff membrane (Elema-Schönander EMT 35, Siemens-Elema) for recording the airway pressure. A gas sampling device was also built into the connector for nitrogen concentration analysis in a nitrogen analyzer (model 47302A; Hewlett-Packard Co., Palo Alto, CA). The analyzer has a sample flow rate of $30 \mathrm{~mL} / \mathrm{min}$ and a rise time of $30 \mathrm{~ms}$.

Measuring procedure. The whole measuring procedure takes place in the infant's incubator. Before the start of each measuring procedure, the general condition, body temperature, heart rate, and blood pressure of the baby are checked, and blood gases are analyzed. The infant is initially placed on the separate bottom plate of the plethysmograph. The cover hood is gently put over the infant, and cables and catheters are pulled through the airtight openings in the hood wall. Remote injection catheters are used 
to ensure blood gas sampling and i.v. administration of drugs and fluids during the study.

The cover hood and bottom plate are then made airtight in relation to one another. While the cover hood is being put over the baby, the ventilator has to be disconnected for a few seconds. The separate face cuff is placed in a circular opening in the cover hood and is then connected firmly to it. The soft face cuff is modeled around the face of the infant. Putty is used to ensure the seal between face and cuff. Air is evacuated from the cuff during modeling and sealing. If necessary, free access to the baby can be obtained in a few seconds by detachment of the parts of the box.

The box is checked for gas leakage before each measurement sequence in the following way: during a short apnea of about 1 $\mathrm{s}$, induced by switching the ventilator to the continuous positive airway pressure mode, a constant gas flow of $50-100 \mathrm{~mL} / \mathrm{s}$ is brought into the box through a separate opening while the outflow through the pneumotachograph is recorded. An identical flow is then led through the pneumotachograph into the box and, if the two recordings differ less than $5 \%$, the system is considered airtight.

The endotracheal tube connector is inserted between the endotracheal tube and the ventilator. The ventilator port not in use is closed with a clamp. A similar ventilator with the same settings is connected to the clamped port. Switching between the two ventilators during pressure and flow recordings then makes it possible to adjust the ventilators to identical settings. The port to the second ventilator is reclamped, and the oxygen concentration is set at $100 \%$, the concentration that is used during the nitrogen washout study.

Before pressure and flow are recorded, infants breathing actively against the ventilator are given a muscle relaxant, (vecuronium bromide $0.1 \mathrm{mg} / \mathrm{kg}$ ). During prolonged studies, this dose can be repeated. When signals on the polygraph are stable, flow and pressure signals from at least 20 continuous breathing cycles are recorded on tape for computer calculations of parameters of ventilation and mechanics of breathing. Zero levels of flow and pressure are also recorded intermittently during the measurements to ensure signal quality during the analyses.

To initiate the washout, the oxygen concentration of ventilatory gas flow is set at $100 \%$ during an expiration. This is done manually by rapidly switching ventilators, using two clamps. During the washout, flow and nitrogen concentrations are re-

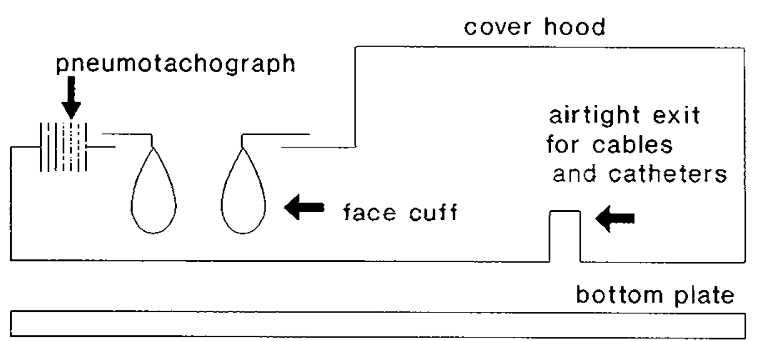

Fig. 1. The body plethysmograph.

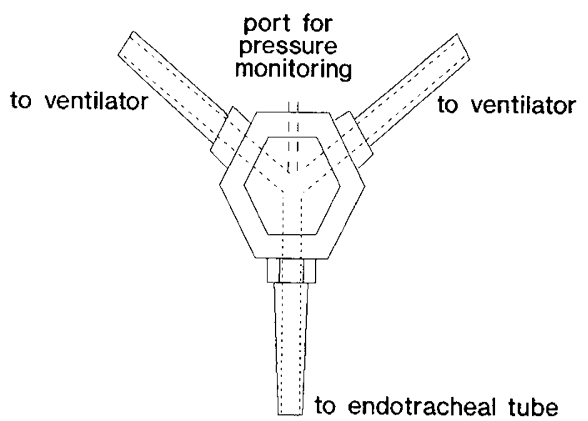

corded on polygraph and tape. When the end-expiratory nitrogen concentration is below $2 \%$, the ventilators are switched back again.

Recording system properties and computer calculations. A personal computer (Digital Professional 350; Digital Equipment Corp., Maynard, MA) was used to analyze the flow, pressure, and nitrogen signals. The analog signals were played back from a tape recorder, filtered by a 4 th order Butterworth low pass filter with a cut-off frequency of $40 \mathrm{~Hz}$ and analog to digital converted using eight-bits resolution. The sampling frequency was $100 \mathrm{~Hz}$ for signals used in estimation of respiratory mechanics and 200 $\mathrm{Hz}$ for nitrogen washout data. The pressure and flow recording systems were matched in phase and amplitude by oscillating flows up to $10 \mathrm{~Hz}$.

The combined effect of the flow resistance of the pneumotachograph and the gas compression in the plethysmograph is one of low pass filtering to the plethysmograph recording system. The time constant of the mechanical system was determined by using two pneumotachographs and a loudspeaker as a volume displacement generator. One of the pneumotachographs recorded the flow from the volume displacement generator and the other, the flow as recorded by the plethysmograph. A body phantom was used to reduce the volume in the plethysmograph appropriately during these measurements. The loudspeaker generated a sinusoidal volume displacement signal at different frequencies from 1 to $8 \mathrm{~Hz}$.

A computer-based algorithm compensated the pneumotachograph flow signal for the bandwidth-limiting effects of the plethysmograph before analysis of respiratory mechanics or nitrogen washout data (1). The conventional single-compartment lung model with two parameters, compliance and resistance, was used to describe the respiratory system mechanics (2). The resistance and compliance parameters were derived for full single breaths as well as for the inspiratory and expiratory phases separately. A least mean square technique was used to estimate the parameters $(1,3,4)$. For comparison, a computer routine derived the compliance at zero flow points and the resistance at midvolume values in the traditional manner (5). The respiratory rate, tidal volume, minimum and maximum values of flow and pressure, mean pressure, and quotient between inspiratory and expiratory time were also derived.

FRC, effective dead space, and nitrogen elimination pattern were calculated from nitrogen washout data according to Sandberg et al. (6). No correction was made for dissolved tissue nitrogen (7).

The accuracy of the volume measurements was determined in a mechanical lung model by comparing the nitrogen washout method with the gas dilution technique. In the lung model, we also investigated whether different baseline gas mixtures and different concentrations of oxygen in the washout gas influenced the accuracy of the volume measurements. A nitrogen washout using an $\mathrm{FiO}_{2}$ of 1.0 was performed after ventilation of the model with an $\mathrm{FiO}_{2}$ of 0.8 . The same procedure was performed with a washout gas with an $\mathrm{FiO}_{2}$ of 0.4 after ventilation of the model with an $\mathrm{FiO}_{2}$ of 0.21 . The results of these two series were

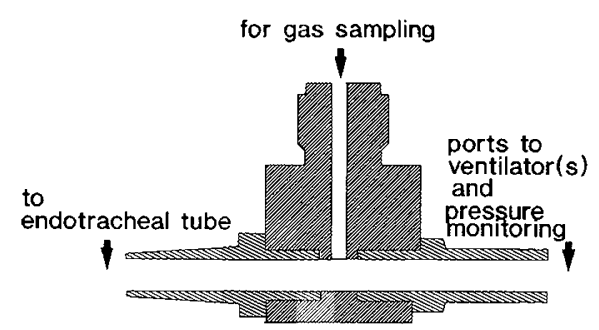

Fig. 2. The endotracheal tube connector. 
compared with a series of washouts with $\mathrm{FiO}_{2} 1.0$ and a ventilatory $\mathrm{FiO}_{2}$ of 0.21 . Ten washouts with each gas combination were performed.

The reproducibility of the measuring procedure was checked by comparing compliance and resistance in a sequence of five breaths with corresponding values from a consecutive sequence of another five breaths. The reproducibility of the nitrogen washout measurements was analyzed in two ways: by repeated measurements in a group of infants at 5 -min intervals and by serial determinations of the volume of an inflatable lung model.

Subjects. Using the method described, we investigated 14 very low birth weight infants with hyaline membrane disease during intermittent positive pressure ventilation within their first $5 \mathrm{~d}$ of life. There were six boys and eight girls. Their median birth weight was $1.29 \mathrm{~kg}$ (range $1.00-1.50 \mathrm{~kg}$ ), and their median gestational age was $29 \mathrm{wk}$ (range 26-33 wk). Each infant was studied after parental consent had been obtained. The project was approved by the ethics committee of Göteborg University. Lung function data from these infants were presented separately (8).

\section{RESULTS}

The measuring procedures, including studies of up to $6 \mathrm{~h}$, were successfully carried out with no need to interrupt for technical or medical complications. The equipment proved safe and functioned well. Occasional air leaks, usually between face and cuff, were easy to detect and correct by remodeling the cuff and by using putty for improved sealing. The method was easy and convenient for the investigators to use, and the time required for a single occasion of measurement was about $30 \mathrm{~min}$. The procedures were also performed without interfering with infant care. Table 1 shows that the infants were investigated without signs of clinical deterioration. In some infants, the ventilator settings were adjusted because of the blood gases analyzed before the start of the measuring procedure. This explains the wide range in some variables in Table 1.

The time constant of the plethysmograph was measured to be $16 \mathrm{~ms}$. When this value was introduced into the flow compensation equation, the compensated flow was found to correspond, in phase and amplitude, to the input signal in the test setup. Therefore, appropriate compensation could be calculated for the filtering effect of the plethysmograph on the measured flow.

A comparison of the mechanical parameters in the subjects, calculated in the traditional manner and using the least mean square method, presented a mean difference of $2.3 \mathrm{~cm} \mathrm{H}_{2} \mathrm{O} / \mathrm{L} / \mathrm{s}$ (SD 5.3) for resistances between 38 and $176 \mathrm{~cm} \mathrm{H}_{2} \mathrm{O} / \mathrm{L} / \mathrm{s}$ and $0.0056 \mathrm{~mL} / \mathrm{cm} \mathrm{H}_{2} \mathrm{O}$ (SD 0.042) for compliances between 0.15 and $1.03 \mathrm{~mL} / \mathrm{cm} \mathrm{H}_{2} \mathrm{O}$. The reproducibility of the least mean square derived parameters is presented in Table 2.

Mean values from repeated determinations of FRC in the sick infants $(n=14)$ differed by $0.4 \mathrm{~mL}$ (SD $2.7 \mathrm{~mL})$. Results from the test of the accuracy of the FRC method are presented in Table 3.

In the mechanical lung model, a washout $\mathrm{FiO}_{2}$ of 0.4 and a breathing gas with room air gave the same mean FRC, $48.4 \mathrm{~mL}$,

Table 1. Body temperature and arterial blood gases in 14 infants before and after examination in body plethysmograph*

\begin{tabular}{lcccc}
\hline \multicolumn{1}{c}{ Measurement } & Before & After & Difference & Range \\
\hline T rect $\left({ }^{\circ} \mathrm{C}\right)$ & 36.9 & 36.5 & 0.4 & $0.9-1.3$ \\
$\mathrm{~T}$ skin $\left({ }^{\circ} \mathrm{C}\right)$ & 36.9 & 36.7 & 0.2 & $-1.2-1.4$ \\
$\mathrm{pH}$ & 7.38 & 7.40 & -0.02 & $-0.17-0.04$ \\
$\mathrm{PCO}_{2}(\mathrm{kPa})$ & 5.4 & 4.8 & 0.6 & $-0.7-4.4$ \\
$\mathrm{HCO}_{3}^{-}(\mathrm{mmol} / \mathrm{L})$ & 23 & 22 & 0.4 & $-3-5$ \\
$\mathrm{PO}_{2}(\mathrm{kPa})$ & 9.9 & 8.2 & 1.6 & $-3-7.6$ \\
$\mathrm{SO}_{2}(\%)$ & 90 & 89 & 0.9 & $-28-11$ \\
\hline
\end{tabular}

* $\mathrm{T}$ rect, rectal temperature; $\mathrm{T}$ skin, skin temperature; $\mathrm{HCO}_{3}^{-}$, bicarbonate ion concentration of plasma; $\mathrm{SO}_{2}$, saturation of $\mathrm{Hb}$ with oxygen.
Table 2. Reproducibility of mechanical parameters in sick newborn infants $(n=14)^{*}$

\begin{tabular}{ccc}
\hline Measurement & $\begin{array}{c}\mathrm{Crs} \\
\left(\mathrm{mL} / \mathrm{cm} \mathrm{H}_{2} \mathrm{O}\right)\end{array}$ & $\begin{array}{c}\mathrm{Rrs} \\
\left(\mathrm{cm} \mathrm{H} \mathrm{H}_{2} \mathrm{O} / \mathrm{L} / \mathrm{s}\right)\end{array}$ \\
\hline 1st observation & 0.46 & 92 \\
2nd observation & 0.47 & 90.3 \\
Mean difference & -0.006 & 1.7 \\
$\mathrm{SD}$ & 0.017 & 2.9 \\
\hline
\end{tabular}

* Crs, compliance of the respiratory system; Rrs, resistance of the respiratory system.

Table 3. Comparison of two methods for determining volume of test lung*

\begin{tabular}{lcc}
\hline \multicolumn{1}{c}{ Method } & Mean & SD \\
\hline Gas dilution $(\mathrm{mL})$ & 51.8 & 1.4 \\
Nitrogen washout $(\mathrm{mL})$ & 50.7 & 1.8 \\
Difference $(\mathrm{mL})$ & 1.1 & \\
\hline
\end{tabular}

* Ten tests were made with each method.

with a coefficient of variation of $2.31 \%$, as compared with 48.4 $\mathrm{mL}$ and $1.93 \%$, respectively, when an $\mathrm{FiO}_{2}$ of 1.0 was used for washout. Washout from a breathing $\mathrm{FiO}_{2}$ of 0.8 resulted in an FRC of $48.5 \mathrm{~mL}$ with a coefficient of variation of $2.76 \%$.

\section{DISCUSSION}

There is renewed interest in lung function measurements in newborn infants with the emergence of commercially available computer techniques and simpler occlusion and inflation methods to assess mechanics of breathing. All of these methods use direct measurement of breathing flow. This is a disadvantage and a serious source of error when measurements are made on intubated infants, as leakage outside the endotracheal tube is a common and unpredictable phenomenon. This makes the flow recorded in the tube differ from the true ventilatory flow. Attempts to seal off leakage by applying gentle pressure on the trachea of the infant may not only disturb the infant but also change the ventilation and, thereby, the ventilatory state of the infant. Another problem with direct flow measurements has to do with the physical dimensions of the pneumotachograph. The common devices commercially available will add a considerable amount of extra dead space, because of the size needed to attain linearity of the flow measurement within the desired range and to keep resistance low. To minimize the added dead space, other types of pneumotachographs with reduced dead space and resistance have been designed (9).

Encouraged by our previous experiences with face-out whole body plethysmography in spontaneously breathing infants (1), we decided to modify this method for mechanically ventilated preterm infants in intensive care. Our new idea was to mount the box around the baby rather than putting the baby into the box. As demonstrated, all infants studied tolerated the investigative procedure well with no alterations in body temperature, circulatory parameters, or blood gases. Extended measurements, over a period of several hours, could be performed without strain on the infants or the investigators. In infants who breathed actively against the ventilator, a muscle relaxant was used as part of our clinical routine to minimize the risks of inadequate ventilation, interstitial pulmonary air leak, and pneumothorax (10). In quiet babies without spontaneous respiratory efforts during the mechanical ventilation, muscle relaxants were not required.

Possible sources of error in flow measurements with volume displacement body plethysmographs are "filtering effects" due to gas compression in the plethysmograph and in lung gas. The effect of the plethysmograph can readily be compensated for as soon as its time constant has been determined, as was found in this work and elsewhere (1). The alveolar gas compression during 
breathing that makes the change in body volume differ from flow at the airway opening is a function of airway resistance, lung volume, and breathing frequency (11). Because these properties can be estimated, it is possible to evaluate the effects of alveolar gas compression upon the flow measurements and compensate for them. We compared resistance and compliance values before and after compensation from 101 corresponding sets of data on lung mechanics and lung volumes. Resistance decreased by a mean value of $11.2 \%$ (SD $4.3 \%$ ), and compliance increased by a mean value of $6.0 \%$ (SD 3.1\%). Thus, the contribution from alveolar gas compression to estimated resistance and compliance values is typically small and has been neglected.

Using the method described, we estimated the mechanical parameters of the respiratory system instead of only those of the lung. The difference can be described as the properties of the chest wall. To distinguish between the mechanical parameters of the lung and the chest wall, esophageal pressure measurements for estimation of intrapleural pressure have been used. This is not considered reliable in preterm neonates with diseased lungs $(12,13)$. Gerhardt and Bancalari (14) measured chest wall compliance $(\mathrm{Ccw})$ in premature infants and found it to vary from $7.2 \mathrm{~mL} / \mathrm{cm} \mathrm{H} \mathrm{H}_{2} \mathrm{O}$ at $28 \mathrm{wk}$ of gestational age to $3.9 \mathrm{~mL} / \mathrm{cm} \mathrm{H}_{2} \mathrm{O}$ at term (14). These values are about $1 / 10$ or less of the dynamic compliance of the whole respiratory system found in our group of infants. Because $1 / \mathrm{Crs}=1 / \mathrm{C}_{L}+1 / \mathrm{Ccw}$, where $\mathrm{Crs}$ is respiratory system compliance and $C_{L}$ is lung compliance, it is evident that Crs under these circumstances is a close estimate of lung compliance in relaxed preterm infants. Chest wall resistance is also only a small fraction of pulmonary resistance, which can consequently be estimated with marginal error by using the resistance of the respiratory system.

Although it has rarely been measured in neonatal intensive care, FRC is a potentially useful variable in the study of lung pathophysiology, because it is a function of lung size, structure, and mechanical properties. FRC is also important for the interpretation of the mechanical parameters. Information of potential importance for the understanding of lung disease in very premature infants may also be derived from analysis of the nitrogen washout pattern. The efficiency of the gas mixing function in the lungs may be assessed and described in terms of effective dead space $(7,15)$, nitrogen clearance $(7)$, or different indices of "distribution of ventilation" $(15,16)$, and alveolar ventilation can be estimated at the same time. (6).

The nitrogen washout method was found to be accurate in the mechanical lung model test as well as in vivo. We also showed, in a lung model, that FRC can be accurately assessed when the $\mathrm{FiO}_{2}$ in the breathing gas is as high as 0.8 . This was also the case in air breathing with a washout $\mathrm{FiO}_{2}$ of only 0.4 . These tests indicate that the method can be used in infants with very high oxygen demand, whereas in infants with less oxygen demand the possible hazards of hyperoxia can be avoided by reducing the oxygen content in the washout gas.

In conclusion, face-out body plethysmography offers a clinically safe method of assessing lung function in mechanically ventilated very preterm newborn infants. It circumvents the problem of leaking endotracheal tubes that afflicts the accuracy of measurement of the mechanics of breathing without increasing dead space. It may also be used together with the nitrogen washout method to assess lung volume, alveolar ventilation, and efficiency of ventilation in critically ill newborns.

\section{REFERENCES}

1. Hjalmarson O 1974 Mechanics of breathing in newborn infants with pulmonary disease. II. Theoretical considerations and methods. Acta Paediatr Scand [Suppl] 247:6-25

2. Mead J, Milic-Emili J 1963 Theory and methodology in respiratory mechanics with glossary of symbols. In: Fenn WO, Rahn H (eds) Handbook of Physiology Section 3: Respiration I. American Physiological Society, Washington, DC, pp 363-376

3. Uhl RR, Lewis FJ 1974 Digital computer calculation of human pulmonary mechanics using a least square fit technique. Comput Biomed Res 7:489495

4. Wald A, Jason D, Murphy TW, Mazza VDB 1969 A computer system for respiratory parameters. Comput Biomed Res 2:411-429

5. Krieger I 1963 Studies on mechanics of respiration in infancy. Am J Dis Child 105:439-448

6. Sandberg K, Sjöqvist BA, Hjalmarson O, Olsson T 1984 Analysis of alveolar ventilation in the newborn. Arch Dis Child 59:542-547

7. Sjöqvist BA, Sandberg K, Hjalmarson O, Olsson T 1986 Method for analyzing multiple breath nitrogen washouts. Med Biol Eng Comput 24:83-90

8. Edberg KE, Sandberg K, Silberberg A, Ekström-Jodal B, Hjalmarson O 1991 Lung volume, gas mixing, and mechanics of breathing in mechanically ventilated very low birth weight infants with idiopathic respiratory distress syndrome. Pediatr Res 30:496-500

9. Brady JP, Deming DD, McCann EM 1985 Neonatal endotracheal flowmeter for tidal volume, airway pressure and end-tidal gas. J Appl Physiol 58:10231025

10. Greenough A, Morley C, Davis J 1983 Interaction of spontaneous respiration with artificial ventilation in preterm babies. J Pediatr 103:769-773

11. Jaeger MJ, Otis AB 1964 Effects of compressibility of alveolar gas on dynamics and work of breathing. J Appl Physiol 19:83-91

12. Thomson A, Elliot J, Silverman M 1983 Pulmonary compliance in sick low birthweight infants. How reliable is the measurement of oesophageal pressure? Arch Dis Child 58:891-896

13. Heaf DP, Turner H, Stocks J, Helms P 1986 The accuracy of oesophageal pressure measurements in convalescent and sick intubated infants. Pediatr Pulmonol 2:5-8

14. Gerhardt T, Bancalari E 1980 Chest wall compliance in full-term and premature infants. Acta Paediatr Scand 69:359-364

15. Cumming G 1967 Gas mixing efficiency in the human lung. Respir Physiol 2:213-224

16. Fowler WS, Cornish Jr ER, Kety SS 1952 Lung function studies. VIII. Analysis of alveolar ventilation by pulmonary $\mathrm{N}_{2}$ clearance curves. J Clin Invest $31: 40-50$ 